\title{
The Value of Titanium Mesh in Cranio-Maxillofacial Reconstructive Surgery
}

\author{
VICTOR VLAD COSTAN ${ }^{1}$, OTILIA BOISTEANU2**, DANIEL TIMOFTE ${ }^{3 * *}$, MARIUS DABIJ A \\ IUniversity of Medicine and Pharmacy Grigore T. Popa, Department of Oral and Maxillofacial Surgery, 16 Universitatii Str., 700115, \\ Iasi, Romania \\ ZUniversity of medicine and Pharmacy Grigore T. Popa, Department of Anesthesiology, 16 Universitatii Str., 700115, Iasi, Romania \\ UUniversity of medicine and Pharmacy Grigore T. Popa, Department of Surgery, 16 Universitatii Str., 700115, lasi, Romania \\ ${ }^{4}$ University of medicine and Pharmacy Grigore T. Popa, Department of Neurosurgery, 16 Universitatii Str., 700115, Iasi, Romania
}

Cranio-maxillofacial defects are often challenging to reconstruct due to the difficulty of selecting the most appropriate material for the individual case while ensuring good outcomes with minimal complications. The chemical properties of titanium regarding biocompatibility coupled with the physical properties of the mesh design, make titanium mesh an optimal material that is increasingly used in reconstructive surgery. We present our experience regarding the use of titanium mesh for the reconstruction of 14 cranio-maxillofacial bone defects of various etiologies, including 8 defects following oncologic resection, 4 posttraumatic sequelae defects, one osteofascial flap donor site defect and one defect following treatment for osteomyelitis. The advantages and disadvantages of this reconstructive method are discussed.

Keywords: titanium mesh, reconstruction, defect, flap, skull base, orbit, sequelae

Titanium is already a well-established material in the medical field, particularly in the area of reconstructive surgery. The main chemical properties that render titanium as an optimal component of medical implantable devices are its corrosion resistance, responsible for the high biocompatibility, and the balance between strength and density which results in a lightweight material that is also resistant. Commercially available titanium is an alloy with the chemical structure Ti-6Al-4V (6\% aluminium, $4 \%$ vanadium) or Ti-6Al-7Nb (6\% aluminium, $7 \%$ niobium) [1]. It is produced in various geometric shapes and sizes, in the form of implants, prostheses and meshes, suitable for different body regions and various types of pathologies.

The titanium mesh is of particular interest for reconstructive surgery due to its wide surface, strength, flexibility and adaptability, which make it highly suitable for a great variety of defects, notably in anatomical regions with a complex geometry, like the cranio-maxillofacial territory. Its current uses are expanding, with more and more studies describing the indications and benefits of its employment in different clinical scenarios [2-7].

The aim of this study is to demonstrate our experience regarding the broad applicability of titanium mesh in the reconstruction of cranio-maxillofacial defects of different etiologies.

\section{Experimental part}

We reviewed 14 cases in which titanium mesh (Fig. 1) was used for the reconstruction of cranio-maxillofacial bone defects. Information was gathered regarding the etiology and location of the defects, the indication for titanium mesh reconstruction, the time of the reconstruction, the challenges encountered during the surgery, as well as the postoperative outcomes and complications. The minimum follow-up period was 6 months.

\section{Results and discussions}

Among the 14 patients, there were 5 women and 9 men, aged between 23 and 86 years old. The defects were due

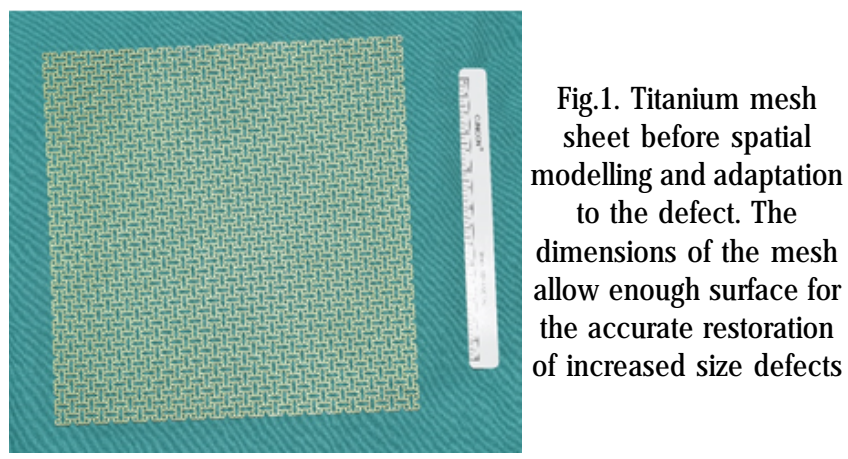

to the ablation of malignant tumors in 8 cases, posttraumatic sequelae in 4 patients, sequelae following treatment for maxillary osteomyelitis in one case, and one defect involved the donor site of a raised osteofascial flap. The bone defects were located in the midface region in 8 cases, the lateral skull base in 3 cases, the glabella region in one patient (Fig. 2, 3), the frontal-orbital region in one case and the parietal skull in one patient. An orbital exenteration had been performed for six patients. The

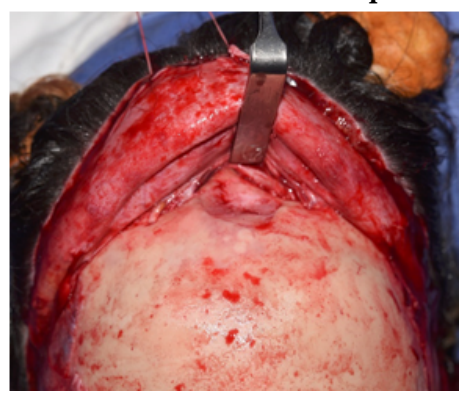

Fig. 2. Exposure of a glabella defect through a coronal

access. The defect was due to an initially untreated nasofronto-orbital fracture

reconstruction of the postablational defects was performed at the time of tumor removal in seven cases, while in one patient the titanium mesh insertion was achieved six months after the tumor removal due to the presence of wound dehiscence and lack of support for the soft tissues. For the posttraumatic cases, the titanium mesh was inserted between six months and one year after the initial trauma. The defect at the flap donor site was repaired one 


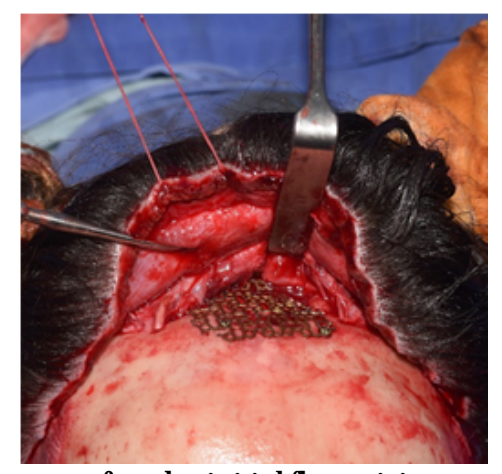

Fig. 3. Titanium mesh reconstruction of the glabella defect showing accurate reconstruction of the bone surface contour

year after the initial flap raising surgery. The defect following osteomyelitis was closed three months after the initial treatment. In seven cases of oncologic defects an additional flap was needed for soft tissue coverage, including four pedicled frontal flaps, one temporalis muscle flap, one pedicled great pectoralis flap and one latissimus dorsi flap. A frontal flap was performed for covering the titanium mesh and soft tissue reconstruction in the case of sequelae following treatment for osteomyelitis.

The titanium mesh was adapted to reconstruct the natural contours of the involved region, or to offer proper support for the overlying flaps used to restore the continuity of the soft tissues. Intraoperatively, we focused on ensuring stability by using at least three points of fixation in a tripod disposition, while also avoiding sharp edges or abrupt corners, especially under thin tissues, like mucosa or the periorbital and nasal skin. The structure and pattern of the mesh offers great freedom in establishing the fixation sites. Another advantage of the mesh pattern is that it offers anchoring points for performing soft tissue suspension using sutures [3]. This method was implemented in one of the posttraumatic sequelae cases for improving the inferior eyelid ectropion. The multiple holes in the mesh allow for drainage of the accumulated blood, a property that is convenient in orbital wall reconstruction, decreasing the risk of developing a postoperative retrobulbar hematoma.

The outcomes of titanium mesh reconstruction were considered favorable in all cases, regarding appearance and function restoration. The mesh served the purpose of the reconstruction in each situation. No infectious complications were noted during the follow-up period. There was no wound dehiscence with mesh exposure. In two patients, the pattern of the mesh was palpable, butno exposure occurred. In one of the postablational cases, the titanium mesh was successfully used for the treatment of a paranasal wound dehiscence, by offering a stable support for the overlying frontal flap, with no further complications. In contrast to our results, one study found wound dehiscence and exposure to be the most prevalent complication of titanium mesh cranioplasty, particularly in patients with general comorbidities or previous surgeries. The same study presents the lowest infection rates for titanium mesh compared to autologous bone graft and polymethyl methacrylate (PMMA) reconstruction [8]. Due to the characteristics of titanium mesh regarding biocompatibility, and the overall low reported infection rates, we successfully used this material for the reconstruction of a defect following treatment for osteomyelitis.

The modelling of the mesh was most important in the posttraumatic sequelae patients, where the aesthetic demands were high. We considered this material to be most useful in the recontouring of the intricate geometry of the midface, including the orbital rims and walls. Good aesthetic results were thus achieved with proper restoration of facial symmetry. Other authors also recognize the benefits of using titanium mesh in regions of complex anatomical shapes, like the zygomato-orbital region $[2,6$, 9]

Many studies underline the suitability of titanium mesh for the reconstruction of exenteration defects and for offering support for local, regional or distant flaps used to reconstruct the regional soft tissues following oncologic resections in the orbito-zygomatic region [2,3,7,11-13]. In our study, we also had good results by inserting titanium mesh for orbito-zygomatic defects following oncologic resection. Optimal defect restoration was achieved by associating titanium mesh for support with a soft tissue flap for restoring skin continuity. One advantage of this association is the addition of quality soft tissues and little to no wound tension, contributing to the absence of dehiscence and mesh exposure.

The advantages of using titanium mesh for skull and skull base reconstruction are outlined by many authors [ 5 , 14-17], along with the low infection rate $[5,8,18]$, placing titanium mesh at the top of the reconstructive preferences for many surgeons. In our experience, using titanium mesh for cranial defects, we obtained proper support, but also protection of the intracranial space from external forces. Additionally, it allowed separation of the reconstructive layers, with less movement and friction of the superficial tissues on the soft intracranial tissue, a benefit that was also mentioned in other studies $[4,15]$.

Due to the high biocompatibility, corrosion resistance, low mass, high stability and resistence coupled with flexibility, malleability, and an overall low infection rate, titanium mesh has become the material of choice for many reconstructive procedures in the cranio-maxillofacial territory $[1,2]$.

\section{Conclusions}

In our experience, titanium mesh is a valuable reconstructive material for the restoration of bone defects involving anatomically complex regions of the cranio-facial skeleton. We obtained favorable outcomes with no infectious complications in the restoration of various defects resulting from different pathologies. We consider that titanium mesh reconstruction is most beneficial for covering extensive bone defects with intricate anatomy, since it can be modelled to accurately reconstruct the geometry of the involved area. It is also useful in rendering support for the overlying soft tissues and in protecting delicate underlying anatomical structures.

\section{References}

1.NEUMANN A, KEVENHOERSTER K. Biomaterials for craniofacial reconstruction. GMS Curr Top Otorhinolaryngol Head Neck Surg. 2009; 8: Doc08.

2.ZHANG WB, YU Y, MAO C, WANG Y, GUO CB, YU GY, PENG $X$. Outcomes of Zygomatic Complex Reconstruction with Patient-Specific Titanium Mesh Using Computer-Assisted Techniques. J Oral Maxillofac Surg. 2019 Mar 23.

3.COSTAN VV, SULEA D, NICOLAU A, DROCHIOI Cl, LUCHIAN ST, BOISTEANU 0 . The use of titanium mesh in facial contour reconstruction. Medical-Surgical J ournal-Revista Medico-Chirurgicala. 2018, 122(1):167-175.

4.DABIJA, M., BOISTEANU, O., DOROBAT, V., DARGOMIR, R., IBRIC, V., COSTAN, V.V., Reconstruction of Skull and Skull Base Defects Using Titanium, Collagen, Polyesterurethane and Other Alloplastic, Allogeneic, Xenogeneic and Autogenic Materials. Rev. Chim. (Bucharest), 69, no.5, 2018, p.1276-1278.

5.KUTTENBERGER JJ, HARDT N. Long-term results following reconstruction of craniofacial defects with titanium micro-mesh systems. J Maxillofac Surg. 2001 Apr;29(2):75-81. 
6.COSTAN VV, DROCHIOI CI, NICOLAU A, SULEA D, DOSCASA, DABIJ A

$M$. Correction of sequelae following orbito-zygomatic fractures. Romanian Journal of Oral Rehabilitation 2018, 10(4):198-206.

7.TARSITANO A, BATTAGLIA S, CIOCCA L, SCOTTI R, CIPRIANI R,

MARCHETTI C. Surgical reconstruction of maxillary defects using a computer-assisted design/computer-assisted manufacturing-produced titanium mesh supporting a free flap. J Craniomaxillofac Surg. 2016 Sep;44(9):1320-6.

8.YEAP MC, TU PH, LIU ZH, HSIEH PC, LIU YT, LEE CY, LAI HY, CHEN CT, HUANG YC, WEI KC, WU CT, CHEN CC. Long-Term Complications of Cranioplasty Using Stored Autologous Bone Graft, ThreeDimensional Polymethyl Methacrylate, or Titanium Mesh After Decompressive Craniectomy: A Single-Center Experience After 596 Procedures. World Neurosurg. 2019 May 10.

9.CIOFU ML, SULEA D, LUCHIAN S, BOISTEANU O. Aesthetic considerations in the reconstruction of orbito-zygomatic fractures. Medical-Surgical J ournal, 2018, 122(1):176-183.

10.COSTAN VV, BOISTEANU O, SULEAD, POPESCU E. The management of skin tumors extended to the orbit. Medical-Surgical J ournal-Revista Medico-Chirurgicala 2018; 122(4):737-743.

11.DEDIOL E, UGLESIC V, ZUBCICV, KNEZEVIC P. Brown class III maxillectomy defects reconstruction with prefabricated titanium mesh and soft tissue free flap. Ann Plast Surg. 2013 Jul;71(1):63-7.
12.COSTAN VV, DRAGOMIR R, COSTEA CF, ROSU A, BOISTEANU O, POPESCU $E$. The ancient indian flap: a technique still up to date. Medical-Surgical J ournal-Revista Medico-Chirurgicala 2018, 122(3): 603613.

13.FU K, LIU Y, GAO N, CAI J, HE W, QIU W. Reconstruction of Maxillary and Orbital Floor Defect with Free Fibula Flap and Whole Individualized Titanium Mesh Assisted by Computer Techniques. J Oral Maxillofac Surg. 2017 Aug; 75(8):1791.e1-1791.e9.

14.COSTAN W, POPESCU E, STRATULAT SI. A New approach to aesthetic maxillofacial surgery: surgical treatment of unilateral exophthalmos due to maxillary sinus muccocele. J Craniofac Surg. May 2013; 24(3):914-916.

15.CHATTOPADHYAY C. Reconstruction of Acquired Frontal Bone Defects Using Titanium Mesh Implants: A Retrospective Study. J Maxillofac Oral Surg. 2019 Mar;18(1):34-39.

16.COSTAN VV, DABIJ A M. Multidisciplinary approach to orbital fracture repair. Romanian Neurosurgery 2018; 32(4): 552-562.

17. HEREDERO JUNG S, DEAN FERRER A, SOLIVERA VELA J, ALAMILLOS GRANADOSF. Spheno-orbital meningioma resection and reconstruction: the role of piezosurgery and premolded titanium mesh. Craniomaxillofac Trauma Reconstr. 2011 Dec;4(4):193-200. 18.LIU JK, GOTTFRIED ON, COLE CD, DOUGHERTY WR, COULDWELL WT. Porous polyethylene implant for cranioplasty and skull base reconstruction. Neurosurg Focus 2004; 16: ECP1.

Manuscript received: 9.01 .2019 